\title{
Sturmian substitutions on two letters, cutting paths and their projections
}

\author{
S. W. Rosema
}

Received: 30 September 2007 / Accepted: 13 May 2008 / Published online: 1 August 2008 (C) The Author(s) 2008

\begin{abstract}
We study the structure of projections of finite cutting paths that correspond to a Sturmian substitution with incidence matrix that has determinant 1 . We show that such projections can be seen as two-letter words, and that there exists another Sturmian substitution that generates these words.
\end{abstract}

Keywords Sturmian substitutions - Cutting path · Lyndon word . Christoffel substitution

Mathematics Subject Classification (2000) Primary 68R15

\section{Introduction}

The history of Sturmian words goes back to Bernoulli in 1772 and Christoffel (1875) [6]. The first in depth study of Sturmian words was made by Morse and Hedlund in 1940 [11]. We call a substitution $\sigma$ over an alphabet of two letters Sturmian if $\sigma$ maps every Sturmian word to a Sturmian word. In 1991 Séébold [17] showed that Sturmian substitutions that have a fixed point are exactly those substitutions that have Sturmian words as fixed points. For further information on Sturmian words and substitutions we refer to Lothaire [9], Chap. 2 and Pytheas Fogg [12], Chap. 6.

If $u=u(0) u(1) \ldots$ is a word defined over the alphabet $\mathcal{A}=\{0, \ldots, n\}$, we define in the $(n+1)$-dimensional space the cutting points corresponding to $u$ by $p_{i}=\left(|u(0) \ldots u(i-1)|_{0}, \ldots,|u(0) \ldots u(i-1)|_{n}\right)$, where $|v|_{a}$ denotes the number of occurrences of the letter $a$ in the word $v$. These cutting points approximate a halfline through the origin, that we call the cutting line, quite well. See Series (1985) [18].

S. W. Rosema $(\bowtie)$

Mathematical Institute, Leiden University, P. O. Box 9512, 2300 RA Leiden, The Netherlands e-mail: swrosema@math.leidenuniv.nl 
We can project the cutting points parallel to the cutting line, onto an $n$-dimensional plane through the origin. In 1982 Rauzy [13] introduced a fractal which is defined as the closure of the projection of the cutting points corresponding to the fixed point of the Tribonacci substitution $0 \rightarrow 01,1 \rightarrow 02,2 \rightarrow 0$. The analogues of this so-called Rauzy fractal have been studied for many other substitutions, see for example [1,2,5,8]. An important question is whether the projection of the cutting points generates a tiling or not. This question was the motivation for Tijdeman and the author [16] to have a closer look at the structure of the projections of the cutting points corresponding to the set of finite words $\sigma^{n}(0)$ in case $\sigma$ is the Fibonacci or Tribonacci substitution. In the Tribonacci case they found a close connection with number systems. This result was generalized by Fuchs and Tijdeman in [7]. In the case of the Fibonacci substitution, Tijdeman and the author projected the cutting points corresponding to $\sigma^{n}(0)$ onto the $y$-axis. By looking at the order of these projected points, they formed a two-letter word $v_{n}$, as we explain in Sect. 3 of this article. They found that the limit word that is generated in this way is a two-sided Fibonacci word. In [15] the author generalized this property to unimodular substitutions defined over two letters. He showed for a special class of Sturmian substitutions, which he called Christoffel substitutions, that the words $v_{n}$ are generated by another Sturmian substitution. For more results on Christoffel substitutions see $[3,4,10]$. In the present paper we generalize the result from [15] to the set of all Sturmian substitutions that have an incidence matrix with determinant 1 .

In Sect. 2 we start with some notation and definitions. Next in Sect. 3 we derive some basic properties of the projections of the cutting points. In Sect. 4 we prove a number of results on Sturmian substitutions which we use in Sect. 5. Finally, in Theorem 5.8 we obtain our main result that the projection words $v_{n}$ are generated by another Sturmian substitution. We give an explicit expression for this substitution in terms of the original substitution.

\section{Notation and definitions}

An alphabet $\mathcal{A}$ is a finite set of elements that are called letters. In this article we always assume $\mathcal{A}=\{0,1\}$. A word is a function $u$ from a finite or infinite block of integers $B$ to $\mathcal{A}$. We call a word $u$ finite when $B$ is finite, infinite when $B=\mathbb{Z}_{\geq 0}$ and bi-infinite when $B=\mathbb{Z}$. If $0 \in B$ we call $u$ a central word. If $k \in B$ and $u(k)=a$ we say $u$ has the letter $a$ at position $k$. When $u$ is a central word, we often underline the letter at position 0 . If $v=v(0) \ldots v(m)$ is a finite word and if $(u(i))_{i \in B}$ is a finite or infinite word, and there exists a $k \in \mathbb{Z}$ such that $v(l)=u(k+l)$ for $l=0, \ldots m$, then $v$ is called a subword of $u$. Moreover, if $k$ is the smallest element of $B$, we say $v$ is a prefix of $u$. On the other hand, if $u$ is finite and $k+m$ is the largest element of $B$, we call $v$ a suffix of $u$. If a word $u$ is finite, we denote by $|u|$ the number of letters in $u$, and by $|u|_{a}$ the number of occurrences of the letter $a$ in $u$.

A word $u$ is called balanced if $\left.|| v\right|_{0}-|w|_{0} \mid<2$ for all subwords $v, w$ of equal length. A finite word $u$ is called strongly balanced if $u^{2}$ is balanced. Here $u^{2}$ is the concatenation of $u$ with $u$. An infinite word is Sturmian if it is balanced and not ultimately periodic. 
We call a finite word $u$ a Lyndon word if every partition $u=v w$ implies $u<w v$ in the lexicographical order.

By cyclically shifting a finite word to the left (right, respectively) we mean removing the right-most (left-most, respectively) letter and placing it on the first open position on the left (right, respectively). By cyclically shifting over a number of positions we mean repeating this procedure a number of times. We call the new word a cyclic shift of the original one.

A substitution $\sigma$ is an application from an alphabet $\mathcal{A}$ to the set of finite words. It extends to a morphism by concatenation, that is, $\sigma(u v)=\sigma(u) \sigma(v)$. It also extends in a natural way to a map from infinite words to infinite words. A substitution over the alphabet $\mathcal{A}$ is primitive if there exists a positive integer $k$ such that, for every $a$ and $b$ in $\mathcal{A}$, the letter $a$ occurs in $\sigma^{k}(b)$. We call $M_{\sigma}:=\left(\begin{array}{ll}|\sigma(0)|_{0} & |\sigma(0)|_{1} \\ |\sigma(1)|_{0} & |\sigma(1)|_{1}\end{array}\right)$ the incidence matrix corresponding to $\sigma$. By $M_{\sigma}^{T}$ we denote the transposed matrix. A fixed point of a substitution $\sigma$ is an infinite word $u$ with $\sigma(u)=u$. For each $a \in \mathcal{A}$ we specify one letter in $\sigma(a)$. If we apply $\sigma$ to a central word $\ldots u(-1) u(0) u(1) \ldots$, then the specified letter of $\sigma(u(0))$ will be the underlined letter at position 0 of $\ldots \sigma(u(-1)) \sigma(u(0)) \sigma(u(1)) \ldots$.

If $x$ is a real number, and $y$ the largest integer that is smaller than or equal to $x$, then we denote by $\{x\}=x-y$ the fractional part of $x$.

\section{Central progressions}

Definition We call a function $w$ a central progression if

- its domain is a block of integers of length $m$ of $\mathbb{Z}$ containing 0 ,

- its image is the set $\{0,1, \ldots m-1\}$,

- there exists a $c \in \mathbb{Z}$ such that if $k$ is in the domain of $w$, then $w(k) \equiv c k(\bmod m)$.

We denote by $|w|$ the length of the interval on which $w$ is defined. Note that it follows from the definition that $\operatorname{gcd}(c, m)=1$.

Example 1 Let the domain of $w$ be $\{-4,-3, \ldots, 1,2\}$, so that $m=7$, and let $c=2$. Then $w(-4)=6, w(-3)=1, w(-2)=3, w(-1)=5, w(0)=0, w(1)=2, w(2)=4$. For convenience we also use the notation $w=6135024$.

Definition Let $u=u(0) \ldots u(m-1)$ be a finite word. The cutting path in the $x$-y-plane corresponding to $u$ consists of $m+1$ integer points $p_{i}$ given by $p_{i}=$ $\left(|u(0) \ldots u(i-1)|_{0},|u(0) \ldots u(i-1)|_{1}\right)$ for $i=0, \ldots, m$, connected by line segments of lengths 1 .

In the sequel of this section we let $u=u(0) \ldots u(m-1)$ denote a finite strongly balanced word containing both zeros and ones, with $\operatorname{gcd}\left(|u|_{0},|u|_{1}\right)=1$. Consider the cutting path corresponding to $u$, and draw the line through the origin and the end point of the path, given by $y=\frac{|u|_{1}}{|u|_{0}} x$. We project each integer point $p_{i}$ on the cutting path parallel to this line onto the $y$-axis. By $P\left(p_{i}\right)$ we denote the second coordinate of the projection of $p_{i}$. It is clear that $P\left(p_{0}\right)=P\left(p_{m}\right)=0$. 


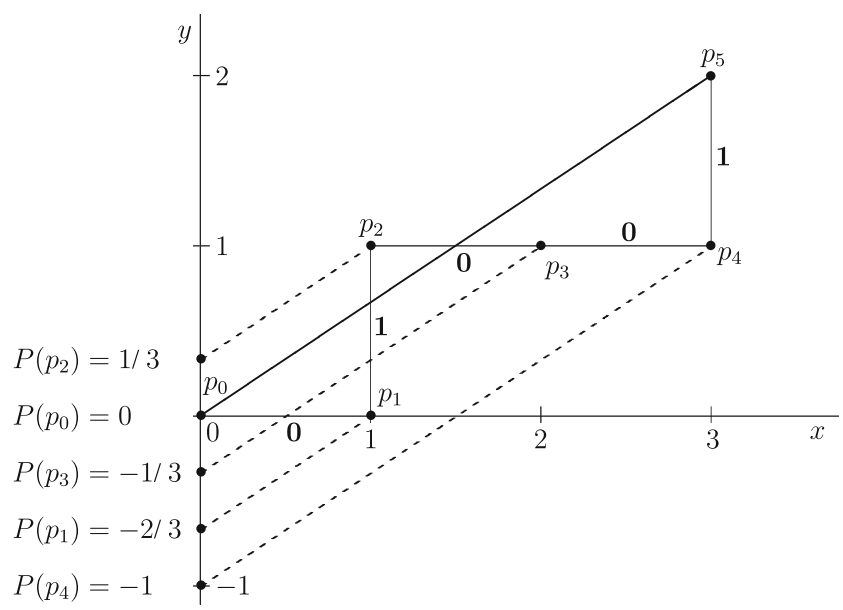

Fig. 1 Projecting the word $u^{(3)}=01001$ leads to the word $w^{(3)}=41302$

Let

$$
D_{u}=\left\{-|u|_{0} P\left(p_{i}\right) \mid i \in\{0, \ldots, m-1\}\right\} .
$$

Note that $D_{u}$ is a set of integers. We define $w_{u}: D_{u} \rightarrow\{0, \ldots, m-1\}$ as follows. If $P\left(p_{i}\right)=k /|u|_{0}$ then $w_{u}(-k)=i$. We say $w_{u}$ has the number $i$ at position $-k$.

Example 2 Let $u=01001$. Then we find that $D_{u}=\{-1,0,1,2,3\}$ and $w_{u}=$ 2031 4, as can be seen in Fig. 1.

Lemma 3.1 For $k \in D_{u}$ we have $w_{u}(k) \equiv k|u|_{1}^{-1} \equiv-k|u|_{0}^{-1}(\bmod m)$, where the inverse is taken modulo $m$.

Proof The first congruence follows from Lemma 2.3 of [15]. The proof of the second congruence follows from $|u|_{0}+|u|_{1}=m$ and $\operatorname{gcd}\left(|u|_{0},|u|_{1}\right)=1$.

Corollary $3.2 w_{u}$ is a central progression.

Proof Proposition 5.1 of [15] states that $D_{u}$ is a block of integers of length $m$, if and only if $u$ is strongly balanced. Since $P\left(p_{0}\right)=0$ we have $0 \in D_{u}$. The third property of a central progression follows from the previous lemma.

Remark From the construction of the cutting path we see that if $w_{u}(k)=i$ then $w_{u}\left(k+|u|_{1}\right)=i+1$ in case $u(i)=0$, and $w_{u}\left(k-|u|_{0}\right)=i+1$ in case $u(i)=1$. We say that to move from number $i$ to $i+1$ in $w_{u}$ we either jump $|u|_{1}$ positions to the right or jump $|u|_{0}$ positions to the left. The number of positions that we jump to the left or right is called the length of the jump.

From now on until the end of this section, we let $w$ be a central progression of length $m$ and $D=\{d, d+1, \ldots, d+m-1\}$ its domain. If $d<0$ we put $z=w(-1)$, if $d=0$ we put $z=w(m-1)$. 
Definition We define $v_{w}$ as the central word that you get by replacing every number in $w$ smaller than $z$ by 0 , and every other number by 1 .

\section{Lemma $3.3 v_{w}$ is strongly balanced.}

Proof It follows from Lemma 3.1 that if $i \in D$, then $w(i)$ equals 0 if $\{-i z / m\} \in$ $[0, z / m)$, and 1 otherwise. According to [9] Sect. 2.1.2 words defined in this way are strongly balanced (so-called rotation words).

The following result follows from [9] Sect. 2.1.2.

Lemma 3.4 $v_{w}$ is a Lyndon word if and only if $d=0$.

We define $\widehat{w}: \mathbb{Z} \rightarrow\{0,1, \ldots m-1\}$ as follows. For given $k \in \mathbb{Z}$, put $\widehat{w}(k)=w(l)$, where $l$ is such that $k \equiv l(\bmod m)$ and $l \in D$. Similarly $\widehat{v_{w}}$ is defined as the bi-infinite periodic continuation of $v_{w}$.

Lemma 3.5 Put $x=w(1)^{-1}(\bmod m)$ and $y=(w(1) x-1) / m$. Then every subword of $\widehat{v_{w}}$ of length $x$ that starts at a position congruent to $-x$ modulo $m$, contains exactly $y+1$ ones, and every other subword of length $x$ contains exactly $y$ ones.

Proof Note that when we move a position to the right, the value of $w$ increases by $w(1)$ modulo $m$. Because $w(1) x=y m+1$, we see that when we start at position $j$ in $\widehat{w}$, with $j$ not equal to $-x$ modulo $m$, and move $x$ times a position to the right, it happens exactly $y$ times that we move to a value that is lower than the previous one. It follows from the definition of $v_{w}$ that $\widehat{w}(i)>\widehat{w}(i+1) \Longleftrightarrow \widehat{w}(i) \geq \widehat{w}(-1) \Longleftrightarrow \widehat{v_{w}}(i)=1$. Therefore the subword of $\widehat{v_{w}}$ of length $x$ starting at position $j$ contains $y$ ones. When we start in a position equal to $-x$ modulo $m, w$ goes down in value $y+1$ times since the last step is to a position with $w$-value 0 , and therefore the subword of length $x$ contains $y+1$ ones.

Lemma 3.6 Let $w$ be a central progression taking values on -1 and 1 . The difference between the number of $w$-values left of 0 that are larger than $w(1)$ and the number of $w$-values left of 0 that are smaller than $w(-1)$ is 0 or 1 .

Proof It follows directly from the definition of central progression that $w(i+1)-w(i)$ is modulo $m$ constant $w(1)$ for every $i$. Therefore every position $j$ in $w$, for which $w(j)<w(-1)$ has a number directly on the right that is larger than $w(j)$, and hence at least equal to $w(1)$, except for the right-most position. Similarly, every position $j$ in $w$, for which $w(j) \geq w(1)$ has a number directly on the left that is smaller than $w(j)$, and hence smaller than $w(-1)$, except for the left-most position. Hence we can form pairs of neighbouring numbers left of 0 for which the right one is larger than $w(1)$ and the left one smaller than $w(-1)$. Since every number left of 0 is either smaller or larger than $w(1)$ and therefore the only number without a companion is the left-most number in case it is larger than $w(1)$, the lemma follows. 


\section{Sturmian substitutions}

We call a $2 \times 2$-matrix Sturmian if it has determinant equal to \pm 1 and has entries in $\mathbb{Z}_{\geq 0}$. We call a substitution $\phi$ over two letters Sturmian if $\phi(u)$ is a Sturmian word for every Sturmian word $u$. The following proposition follows directly from Proposition 2.3.10 of [9].

Proposition 4.1 A two-letter substitution $\phi$ is Sturmian if and only if there exist two sequences of words $u^{(n)}, v^{(n)}$ such that

- $u^{(0)}=0, v^{(0)}=1$ or $u^{(0)}=1, v^{(0)}=0$,

- for every $n \geq 0$ we have $u^{(n+1)} \in\left\{u^{(n)} v^{(n)}, v^{(n)} u^{(n)}\right\}, v^{(n+1)}=v^{(n)}$ or $u^{(n+1)}=$ $u^{(n)}, v^{(n+1)} \in\left\{u^{(n)} v^{(n)}, v^{(n)} u^{(n)}\right\}$,

- there exists an $m$ such that $\phi(0)=u^{(m)}, \phi(1)=v^{(m)}$.

Lemma 4.2 If $\phi$ is a Sturmian substitution, then $\phi^{n}(0)$ is strongly balanced for every $n>0$.

Proof It follows from Corollary 9 of [14] that a Sturmian substitution maps every finite balanced word to a finite balanced word. Hence $\phi^{n}(0) \phi^{n}(0)=\phi^{n}(00)$ is balanced.

Let $M=\left(\begin{array}{ll}a & b \\ c & d\end{array}\right)$ be a Sturmian matrix. If $a+b>c+d$, then we call $M$ an upper matrix, if $a+b<c+d$ a lower matrix.

Let $u=u(0) \ldots u(m-1)$ denote a finite strongly balanced word containing both zeros and ones with $\operatorname{gcd}\left(|u|_{0},|u|_{1}\right)=1$ and let $x$ be an integer with $0 \leq x<|u|$. Then $w_{u}{ }^{-1}(x)$ denotes the position of the number $x$ in the central progression $w_{u}$.

Lemma 4.3 Let $\phi$ be a Sturmian substitution with as incidence matrix a lower matrix $M_{\phi}=\left(\begin{array}{ll}a & b \\ c & d\end{array}\right)$ with abcd $\neq 0$, and such that $\phi(0)$ is a prefix of $\phi(1)$. Let $x, y$ be two different integers such that $0 \leq x<|\phi(0)|, 0 \leq y<|\phi(0)|$ and $w_{\phi(0)}{ }^{-1}(x)<$ $w_{\phi(0)}{ }^{-1}(y)$. Then $w_{\phi(1)}{ }^{-1}(x)<w_{\phi(1)}{ }^{-1}(y)$.

Proof It suffices to prove the statement for neighbouring numbers $x, y$ in $w_{\phi(0)}$, with $x$ left of $y$. Note that a jump in $w_{\phi(0)}$ to the left has length $a$ and to the right length $b$. Similarly, a jump in $w_{\phi(1)}$ to the left has length $c$ and to the right length $d$.

First assume $x<y$. We call $i, j$ the number of jumps to the left and to the right, respectively, that we have to make in $w_{\phi(0)}$ to get from $x$ to $y$. Hence $j b-i a=1$. It is clear that $j \leq a$ and it follows from [15] Corollary 4.1 that $a \leq c$. Since det $M_{\phi}= \pm 1$ we get $a(j d-i c)=c(j b-i a) \pm j=c \pm j \geq 0$. If $j d-i c=0$, then in $w_{\phi(1)}$ after $j$ jumps to the right and $i$ to the left, we are back in our starting position. Hence $i d-j c>0$. Since $\phi(0)$ is a prefix of $\phi(1)$, it follows that if we go from $x$ to $y$ in $w_{\phi(1)}$ we also make $i$ jumps to the left and $j$ to the right. Hence we move $j d-i c \geq 1$ positions to the right.

Now assume $x>y$. Then we find in the same way that when we go from $x$ to $y$ in $w_{\phi(1)}$ we move at least 1 position to the left. 
Lemma 4.4 Let $\phi$ be a Sturmian substitution with as incidence matrix a lower matrix $M_{\phi}=\left(\begin{array}{ll}a & b \\ c & d\end{array}\right)$ with abcd $\neq 0$, and such that $\phi(0)$ is a suffix of $\phi(1)$. Let $x, y$ be two different integers such that $0 \leq x<|\phi(0)|, 0 \leq y<|\phi(0)|$ and $w_{\phi(0)}{ }^{-1}(x)<$ $w_{\phi(0)}{ }^{-1}(y)$. Then $w_{\phi(1)}{ }^{-1}(x+c+d-(a+b))<w_{\phi(1)}{ }^{-1}(y+c+d-(a+b))$.

Proof The proof is analogous to the proof of the previous lemma.

Lemma 4.5 Let $\left(\begin{array}{cc}a & b \\ c+a g & d+c g\end{array}\right)$ be a Sturmian matrix with determinant 1 , such that $\left(\begin{array}{ll}a & b \\ c & d\end{array}\right)$ is an upper matrix. Then $a^{-1}=c+d(\bmod a+b)$.

Proof This follows directly from the fact that $a d-b c=1$.

Lemma 4.6 Let $\phi$ be a Sturmian substitution that has a fixed point starting with 0 , and has an incidence matrix $M_{\phi}=\left(\begin{array}{ll}a & b \\ c & d\end{array}\right)$ that has determinant equal to 1 . Let $x, y$ be two different integers such that $0 \leq x<|\phi(0)|, 0 \leq y<|\phi(0)|$ and let $n$ be a positive integer so that $w_{\phi^{n}(0)}^{-1}(x)<w_{\phi^{n}(0)}^{-1}(y)$. Then $w_{\phi^{n+1}(0)}^{-1}(x)<w_{\phi^{n+1}(0)}^{-1}(y)$.

Proof It suffices to prove the statement for neighbouring numbers $x, y$ in $w_{\phi^{n}(0)}$, with $x$ left of $y$. Put $\left(\begin{array}{ll}a_{n} & b_{n} \\ c_{n} & d_{n}\end{array}\right):=M_{\phi}^{n}$. Note that a jump in $w_{\phi^{n}(0)}$ to the left has length $a_{n}$ and a jump to the right length $b_{n}$. Since

$$
M_{\phi}^{n+1}=\left(\begin{array}{cc}
a a_{n}+b c_{n} & a b_{n}+b d_{n} \\
\ldots & \ldots
\end{array}\right)
$$

a jump in $w_{\phi^{n+1}(0)}$ to the left has length $a a_{n}+b c_{n}$ and a jump to the right length $a b_{n}+b d_{n}$.

First assume $x<y$. We call $i, j$ the number of jumps in $w_{\phi^{n}(0)}$ to the left and to the right, respectively, that we have to make to get from $x$ to $y$. Hence $j b_{n}-i a_{n}=1$. Since $w_{\phi^{n}(0)}$ is a prefix of $w_{\phi^{n+1}(0)}$, we see that to move from $x$ to $y$ in $w_{\phi^{n+1}(0)}$ we move $j b_{n+1}-i a_{n+1}$ positions to the right. We have

$$
\begin{aligned}
a_{n}\left(j b_{n+1}-i a_{n+1}\right) & =a_{n}\left(a+j b d_{n}-i b c_{n}\right)=a a_{n}+j b+j b b_{n} c_{n}-i b a_{n} c_{n} \\
& =a a_{n}+b c_{n}+j b>0
\end{aligned}
$$

hence the integer $y$ is on the right of $x$ in $w_{\phi^{n+1}(0)}$.

Now assume $x>y$. We call $i, j$ the number of jumps in $w_{\phi^{n}(0)}$ to the left and to the right, respectively, that we have to make to get from $y$ to $x$. Hence $j b_{n}-i a_{n}=-1$. To move from $y$ to $x$ in $w_{\phi^{n+1}(0)}$ we move $j b_{n+1}-i a_{n+1}$ positions to the right. We have

$$
\begin{aligned}
a_{n}\left(j b_{n+1}-i a_{n+1}\right) & =a_{n}\left(-a+j b d_{n}-i b c_{n}\right)=-a a_{n}+j b+j b b_{n} c_{n}-i b a_{n} c_{n} \\
& =-a a_{n}-b c_{n}+j b .
\end{aligned}
$$


If $M_{\phi}^{n}$ is a lower matrix, we have $j \leq a_{n} \leq c_{n}$ which gives $j b_{n+1}-i a_{n+1}<0$ and we are done. Assume $M_{\phi}^{n}$ is an upper matrix. Note that $w_{\phi^{n}(0)}(-1)=c_{n}+d_{n}$, where we use Lemma 3.1 and Lemma 4.5. In case $j \leq c_{n}$ we have $-a a_{n}-b c_{n}+j b<0$ hence $j b_{n+1}-i a_{n+1}<0$ and we are done. In case $j>c_{n}$ we have $i<d_{n}$, hence

$$
b_{n}\left(j b_{n+1}-i a_{n+1}\right)=b_{n}\left(-a+j b d_{n}-i b c_{n}\right)=-a b_{n}+i b-b d_{n}<0,
$$

and thus $j b_{n+1}-i a_{n+1}<0$.

Definition Let $M_{\phi}=\left(\begin{array}{ll}a & b \\ c & d\end{array}\right)$ be a Sturmian matrix with determinant equal to 1 and $a b c d \neq 0$. Let $\phi$ be the substitution that has $M_{\phi}$ as incidence matrix, such that $\phi(0), \phi(1)$ are balanced Lyndon words. Then we call $\phi$ a Christoffel substitution.

It follows from [15] Sect. 6 that for every Sturmian matrix there exists a unique Christoffel substitution that has that matrix as incidence matrix. The following result is proved as Lemma 6.4 of [15].

Lemma 4.7 Let $\phi$ be a Christoffel substitution. If the corresponding incidence matrix is an upper matrix, there exist words $u$ and $v$, possibly empty, such that $\phi(0)=u 0 v$ and $\phi(1)=u 1$. If it is a lower matrix, there exist words $u$ and $v$, possibly empty, such that $\phi(0)=u 0 v$ and $\phi(1)=(u 0 v)^{k} u 1$ for some positive integer $k$.

Corollary 4.8 Let $\phi$ be a Christoffel substitution. If the corresponding incidence matrix is a lower matrix, there exist possibly empty words $u$ and $v$ such that $\phi(0)=0 u$ and $\phi(1)=v 1 u$. If it is an upper matrix, there exist possibly empty words $u$ and $v$ such that $\phi(0)=0 u(v 1 u)^{k}$ and $\phi(1)=v 1 u$ for some positive integer $k$.

Proof Let $\phi$ have incidence matrix $\left(\begin{array}{ll}a & b \\ c & d\end{array}\right)$. Note that if we start with a Lyndon word, interchange the zeros and ones, and read it backwards, the result is a Lyndon word again. Let $\psi$ be the substitution obtained from $\phi$ by interchanging the zeros and ones, swapping $\psi(0)$ and $\psi(1)$ and reading them backwards. Then $\psi$ has incidence matrix $\left(\begin{array}{ll}d & c \\ b & a\end{array}\right)$, and the result follows directly from Lemma 4.7.

Lemma 4.9 Let $\phi$ be a Christoffel substitution that has an upper matrix as incidence matrix. Let $k$ with $0<k<|\phi(0)|$ be an integer and let $\psi$ be the substitution such that you get $\psi(0)$ by cyclically shifting $\phi(0)$ over $k$ positions to the left, and $\psi(1)$ by cyclically shifting $\phi(1)$ over $k$ positions to the left. Then

a) $\psi(1)$ is a prefix of $\psi(0)$,

b) $\psi$ is a Sturmian substitution.

Proof a) It follows from Corollary 4.8 that $\phi(0)=0 u(\phi(1))^{l}$ with $u$ a suffix of $\phi(1)$, and from Lemma 4.7 that $\phi(1)=v 1$ with $v$ a prefix of $\phi(0)$. This implies the statement.

b) First let $\psi$ be the substitution that corresponds to $k=1$. We know from (a) that $\psi(1)$ is a prefix of $\psi(0)$. We use induction to show that $\psi^{n}(0)$ is a cyclic shift of $\phi^{n}(0)$ 
for every $n>0$. For $n=1$ this is clear. Assume it is true for $n=m-1$. Consider the word $\psi^{m}(0)$. By cyclically shifting it one position to the right, we get the word $\phi\left(\psi^{m-1}(0)\right)$, where we use that $\psi(0)$ and $\psi(1)$ start with the same letter. It follows from the induction hypothesis that this is a cyclic shift of $\phi^{m}(0)$.

Now let $k$ be any number with $0<k<|\phi(0)|$ and $\psi$ the corresponding cyclic shift of $\phi$. By induction on $k$ it follows that $\psi^{n}(0)$ is a cyclic shift of $\phi^{n}(0)$ for every $n>0$. Since $\phi$ is a Christoffel substitution, $\phi^{n}(0)$ is strongly balanced for every $n$, and it follows that $\psi^{n}(0)$ is strongly balanced. Hence the limit word that you get by letting $n \rightarrow \infty$ is a Sturmian word that is mapped to itself by $\psi$, and it follows from [9] Theorem 2.3.7 that $\psi$ is a Sturmian substitution.

Proposition 4.10 Let $N=\left(\begin{array}{ll}a & b \\ c & d\end{array}\right)$ be a Sturmian upper matrix with determinant 1 and let $v$ be a strongly balanced word that is not Lyndon, with $|v|_{0}=a,|v|_{1}=b$. Then the substitution $\psi$, with $\psi(0)=v$ and $\psi(1)$ the prefix of $\psi(0)$ of length $c+d$, is a Sturmian substitution that has $N$ as incidence matrix.

Proof Let $k$ be the number of times you have to cyclically shift $v$ to the right, to get a Lyndon word. Let $\phi$ be the Christoffel substitution that has $N$ as incidence matrix. Let $\psi^{\prime}$ be the substitution such that you get $\psi^{\prime}(0)$ by cyclically shifting $\phi(0)$ over $k$ positions to the left, and $\psi^{\prime}(1)$ by cyclically shifting $\phi(1)$ over $k$ positions to the left. Then according to Lemma 4.9 (a) $\psi^{\prime}(1)$ is a prefix of $\psi^{\prime}(0)$, hence $\psi^{\prime}=\psi$. According to Lemma 4.9 (b) $\psi$ is a Sturmian substitution. Clearly $\psi^{\prime}$ has the same incidence matrix as $\phi$ which is $N$.

Remark The result of Proposition 4.10 does not hold if $\psi(0)$ is a Lyndon word. Take for example $N=\left(\begin{array}{ll}3 & 2 \\ 1 & 1\end{array}\right)$ and $\psi(0)=00101$. Then $\psi(1):=00$ is the prefix of $\psi(0)$ of length 2, but the substitution $\psi$ is not Sturmian and does not have $N$ as incidence matrix.

\section{Projecting Sturmian substitutions}

From now on let $M_{\sigma}=\left(\begin{array}{cc}a & b \\ c+a g & d+b g\end{array}\right)$ be a Sturmian matrix with determinant 1 , such that $\left(\begin{array}{ll}a & b \\ c & d\end{array}\right)$ is an upper Sturmian matrix, and let $\sigma$ be a primitive Sturmian substitution that has $M_{\sigma}$ as incidence matrix, has a fixed point starting with 0 , and such that $\sigma(0)$ is not a Lyndon word. Note that the case that $\sigma$ is a Christoffel substitution has been considered in Sect. 6 of [15].

Put $w_{n}=w_{\sigma^{n}(0)}$ and $v_{n}=v_{w_{n}}$ for $n \geq 1$, where $w_{u}$ and $v_{w}$ are defined in Sect. 3. Denote by $e, f$ the number of values left of the 0 position in $w_{\sigma(0)}$ and $w_{\sigma(1)}$ respectively, by $p$ the number of 0 's in $v_{1}$ left of the underlined letter, and set $r=e+b(f-p-e g)$. Note that $e>0$ since $\sigma(0)$ is not a Lyndon word, hence $p<e$ and $r>0$. 
Example 3 Let $M_{\sigma}=\left(\begin{array}{ll}3 & 2 \\ 7 & 5\end{array}\right)$ and $\sigma(0)=01001, \sigma(1)=010010101001$. Then $g=2, w_{\sigma(0)}=20314, v_{1}=1 \underline{0101}$ and $w_{\sigma(1)}=92705103816114$. Hence $e=1, f=3, p=0$ and $r=3$.

Lemma 5.1 Let $g=0$. Then $f-p=0$ or $f-p=1$.

Proof By Proposition 4.1, we know that $\sigma(1)$ is a prefix of $\sigma(0)$, or $\sigma(1)$ is a suffix of $\sigma(0)$.

Assume $\sigma(1)$ is a prefix of $\sigma(0)$. If we apply Lemma 4.3 to the substitution $\phi$ with $\phi(0)=\sigma(1)$ and $\phi(1)=\sigma(0)$, we see that the $c+d$ numbers of $w_{\sigma(1)}$ appear in the same order in $w_{\sigma(0)}$. Hence $f$ equals the number of $w$-values in $w_{\sigma(0)}$ left of 0 that are smaller than $c+d$. It follows from Lemma 3.1 and Lemma 4.5 that $w_{\sigma(0)}(-1)=c+d$. Since $p$ is by definition the number of zeros left of the underlined letter in $v_{1}$, we get $f=p$.

Now assume $\sigma(1)$ is a suffix of $\sigma(0)$. Then the sequence of jumps to the right and left in $w_{\sigma(1)}$ starting from the number 0 , are in the same order as the sequence of jumps to the right and left in $w_{\sigma(0)}$ starting from the number $a+b-(c+d)$. Hence $f$ equals the number of $w$-values in $w_{\sigma(0)}$ left of $a+b-(c+d)$ that are larger than $a+b-(c+d)$. It follows from $w_{\sigma(0)}(-1)=c+d$ that $w_{\sigma(0)}(1)=a+b-(c+d)$. Recall that $p$ equals the number of $w$-values in $w_{\sigma(0)}$ left of 0 that are smaller than $c+d$. It follows from Lemma 3.6 that $f-p$ is 0 or 1 .

Lemma 5.2 If $g>0$, there exist non-negative integers $x, y$ with $x+y=g$ and $a$ word $u$ such that $\sigma(1)=\sigma(0)^{x} u \sigma(0)^{y}$ and such that $u$ is a prefix or suffix of $\sigma(0)$.

Proof Since $g$ is the maximum number of times we can subtract the sum of the entries in the top row of $M_{\sigma}$ from the sum of the entries in the bottom row, it follows from Proposition 4.1 that $g$ is the maximum number of times we can remove $\sigma(0)$ as a prefix or suffix from $\sigma(1)$. It also follows from Proposition 4.1 that $u$ is a prefix or suffix of $\sigma(0)$.

\section{Lemma 5.3 We have}

(i) $(d+b g)^{-1} \equiv a+b(\bmod c+d+a g+b g)$,

(ii) $(c+d)\left(a^{2}+b c+a b g\right) \equiv a+b g\left(\bmod a^{2}+b c+a b g+a b+b d+b^{2} g\right)$,

(iii) $\left(a^{2}+b c+a b g\right)^{-1} \equiv a c+c d+a d g+b c+d^{2}+b d g\left(\bmod a^{2}+b c+a b g+\right.$ $\left.a b+b d+b^{2} g\right)$.

Proof (i) $(a+b)(d+b g)-1=b(c+d+a g+b g)$, for (ii), (iii) the proofs are similar.

Proposition $5.40 \leq f-p-e g \leq g+1$.

Proof In case $g=0$ this follows from Lemma 5.1.

Assume $g>0$. According to Lemma 5.2 there exist non-negative integers $x, y$ with $x+y=g$ and a word $u$ that is a prefix or suffix of $\sigma(0)$ such that $\sigma(1)=$ $\sigma(0)^{x} u \sigma(0)^{y}$. From (i) in Lemma 5.3 it follows that $|\sigma(1)|_{1}^{-1} \equiv|\sigma(0)|(\bmod |\sigma(1)|)$. 
Hence according to Lemma 3.1 we have $w_{\sigma(1)}(1)=|\sigma(0)|=a+b$. So when we start at position 0 in $w_{\sigma(1)}$ and make $a+b$ jumps, where the jumps follow the pattern indicated by the word $\sigma(0)$, we end up at position 1 . After repeating this process $x$ times we end up at position $x$. After making the next $|u|$ jumps we must end up at position $-y$, since after repeating the process of making $a+b$ jumps $y$ times at the end, we get back at position 0 . This yields $y \leq g$ numbers of $w_{\sigma(1)}$ in the negative positions $-y,-y+1, \ldots,-1$.

Notice that $w_{\sigma(0)}$ contains the numbers 0 up to $a+b-1$, of which by definition $e$ numbers are left of 0 . If $w_{\sigma(0)}$ is a prefix of $w_{\sigma(1)}$ (which means $x>0$ ), it follows from Lemma 4.3 that the numbers between 0 and $a+b$ appear in the same order in $w_{\sigma(1)}$ as they do in $w_{\sigma(0)}$. Hence $w_{\sigma(1)}$ has exactly $e$ numbers between 0 and $a+b$ that are left of position $-y$. If $w_{\sigma(0)}$ can be removed twice as a prefix of $w_{\sigma(1)}$ (which means $x>1)$, the jumps from $a+b$ to $2(a+b)$ in $w_{\sigma(1)}$ are an exact copy of the jumps from 0 to $a+b$. So $w_{\sigma(1)}$ also has exactly $e$ numbers between $a+b$ and $2(a+b)$ that are left of position $-y$. Continuing in this way up to $x$ times, this generates exactly $x e$ numbers left of position $-y$ in $w_{\sigma(1)}$.

In the same way, using Lemma 4.4, we see that ye numbers are generated left of position $-y$ in $w_{\sigma(1)}$ by the suffix $\sigma(0)^{y}$ of $\sigma(1)$. Together this gives $x e+y e=e g$ numbers left of position $-y$ in $w_{\sigma(1)}$.

Now we consider the remaining subword $u$ which is a prefix or suffix of $\sigma(0)$. When we jump in $w_{\sigma(1)}$ from position $x$ to position $-y$, where the jumps follow the pattern indicated by the word $u$, it generates a certain amount of values left of position $-y$ in $w_{\sigma(1)}$. We shall show that this amount is equal to $p$ or $p+1$.

Note that $|u|_{0}=c,|u|_{1}=d$. Recall that $w_{\sigma(0)}(-1)=c+d$ and $w_{\sigma(0)}(1)=$ $a+b-(c+d)$. Consider the substitution $\phi$ with $\phi(0)=u$ and $\phi(1)=\sigma(0)$, which has incidence matrix $M_{\phi}=\left(\begin{array}{ll}c & d \\ a & b\end{array}\right)$. It follows from Proposition 4.1 that $\phi$ is a Sturmian substitution.

In case $u$ is a prefix of $\sigma(0)$, we see by applying Lemma 4.3 to $\phi$, that the $c+d$ numbers in $w_{\phi(0)}=w_{u}$ are in the same order as the numbers 0 up to $c+d-1$ in $w_{\phi(1)}=w_{\sigma(0)}$. Hence the number of values left of 0 in $w_{u}$ equals the number of values left of 0 in $w_{\sigma(0)}$ that are smaller than $c+d=w_{\sigma(0)}(-1)$, which is $p$ by definition.

In case $u$ is a suffix of $\sigma(0)$, we see by applying Lemma 4.4 to $\phi$, that the $c+d$ numbers in $w_{\phi(0)}=w_{u}$ are in the same order as the numbers $a+b-(c+d)$ up to $a+b-1$ in $w_{\phi(1)}=w_{\sigma(0)}$. Hence the number of values left of 0 in $w_{u}$ equals the number of values in $w_{\sigma(0)}$ left of $w_{\sigma(0)}(1)=a+b-(c+d)$ that are at least $a+b-(c+d)$. Since $p$ equals by definition the number of values in $w_{\sigma(0)}$ left of 0 that are smaller than $w_{\sigma(0)}(-1)$, it follows from Lemma 3.6 that the number of $w$-values left of 0 in $w_{u}$ is $p$ or $p+1$.

Because the order of the $c+d$ numbers in $w_{\phi(0)}=w_{u}$ is preserved in $w_{\phi(1)}=w_{\sigma(0)}$, and the order of the $a+b$ numbers in $w_{\sigma(0)}$ is preserved in $w_{\sigma(1)}$, we see that when we jump in $w_{\sigma(1)}$ from position $x$ to position $-y$, where the jumps follow the pattern indicated by the word $u$, it generates either $p$ or $p+1$ numbers left of position $-y$ in $w_{\sigma(1)}$. It follows that the total number of values left of position 0 in $w_{\sigma(1)}$ is in between $p+e g+y$ and $p+e g+y+1$, hence in between $p+e g$ and $p+e g+$ $g+1$. 
Definition We denote by $\tau$ the substitution that has

$$
M_{\tau}:=\left(\begin{array}{cc}
1 & g+1 \\
1 & g
\end{array}\right) M_{\sigma}^{T}\left(\begin{array}{cc}
-g & g+1 \\
1 & -1
\end{array}\right)
$$

as incidence matrix, and which is such that

- if we cyclically shift $\tau(0)$ over $r$ positions to the right, we get a Christoffel word,

- the $(r+1)$ th letter of $\tau(0)$ is underlined,

- $\tau(1)$ is a prefix of $\tau(0)$.

It follows from Proposition 4.10 that $\tau$ is a Sturmian substitution.

Example 3 (Continued). We get $M_{\tau}=\left(\begin{array}{ll}4 & 5 \\ 3 & 4\end{array}\right)$ and $\tau(0)=011010101$, $\tau(1)=0110101$. Note furthermore that

$$
\sigma^{2}(0)=010010100101010010100101001010010101001,
$$

$w_{2}=w_{\sigma^{2}(0)}=1436192247291234170225271032153720325830$ 1335181236281133163821426931 and

$$
v_{2}=0110101011 \underline{01010101101010110101010110101 .}
$$

Lemma 5.5 Let $n \geq 1$. Then the number of values left of the 0 position in the central progression $w_{n}$ equals

$$
(1,0) \sum_{k=0}^{n-1} M_{\sigma}^{k}\left(\begin{array}{l}
e \\
f
\end{array}\right)
$$

Proof For $n=1$ the statement follows directly from the definition of $e$. Assume the statement is true for some positive integer $n$.

According to Lemma 4.6 the numbers in $w_{n}$ appear in the same order in $w_{n+1}$. Put

$$
\begin{aligned}
M_{\sigma}= & \left(\begin{array}{ll}
a^{\prime} & b^{\prime} \\
c^{\prime} & d^{\prime}
\end{array}\right) \text { and } M_{\sigma}^{n}=\left(\begin{array}{ll}
a_{n}^{\prime} & b_{n}^{\prime} \\
c_{n}^{\prime} & d_{n}^{\prime}
\end{array}\right) \text {. Using } \\
& \left(\begin{array}{ll}
a_{n+1}^{\prime} & b_{n+1}^{\prime} \\
c_{n+1}^{\prime} & d_{n+1}^{\prime}
\end{array}\right)=\left(\begin{array}{ll}
a_{n}^{\prime} & b_{n}^{\prime} \\
c_{n}^{\prime} & d_{n}^{\prime}
\end{array}\right)\left(\begin{array}{ll}
a^{\prime} & b^{\prime} \\
c^{\prime} & d^{\prime}
\end{array}\right)=\left(\begin{array}{ll}
a^{\prime} & b^{\prime} \\
c^{\prime} & d^{\prime}
\end{array}\right)\left(\begin{array}{ll}
a_{n}^{\prime} & b_{n}^{\prime} \\
c_{n}^{\prime} & d_{n}^{\prime}
\end{array}\right)
\end{aligned}
$$

it is easy to check that $a^{\prime} b_{n+1}^{\prime}-b^{\prime} a_{n+1}^{\prime}=b_{n}^{\prime}$ and $c^{\prime} b_{n+1}^{\prime}-d^{\prime} a_{n+1}^{\prime}=-a_{n}^{\prime}$. It follows that if there exists a jump to the right in $w_{n}$ from some position $x$ to some position $y$, then there exists a series of $a^{\prime}+b^{\prime}$ jumps in $w_{n+1}$ from position $x$ to position $y$. Similarly if there exists a jump to the left in $w_{n}$ from some position $x$ to some position $y$, then there exists a series of $c^{\prime}+d^{\prime}$ jumps in $w_{n+1}$ from position $x$ to position $y$.

It follows from the previous remarks, that the first $a^{\prime}+b^{\prime}$ jumps in $w_{n+1}$, from position 0 to position $b_{n}^{\prime}$, generate $e$ numbers that are located left of the most left number in $w_{n}$. In the same way each other jump to the right in $w_{n}$ corresponds to a 
series of $a^{\prime}+b^{\prime}$ jumps in $w_{n+1}$ which each generate $e$ numbers at positions left of the most left position of $w_{n}$, and each jump to the left in $w_{n}$ corresponds to a series of $c^{\prime}+d^{\prime}$ jumps in $w_{n+1}$ which each generate $f$ numbers at positions left of the most left position of $w_{n}$.

Note that the number of jumps to the right and left in $w_{n}$ are given by the two entries in the top row of $M_{\sigma}^{n}$, respectively. Hence $w_{n+1}$ contains the same number of values left of 0 as $w_{n}$ has, augmented with $(1,0) M_{\sigma}^{n+1}\left(\begin{array}{l}e \\ f\end{array}\right)$, and the result follows.

Let $q$ be the number of zeros in $\tau(0)$ left of the underlined letter.

Lemma 5.6 For $n \geq 1$ the number of letters left of the underlined letter in $\tau^{n-1}\left(v_{1}\right)$ equals

$$
\left((p, e-p) \sum_{k=0}^{n-1} M_{\tau}^{k}+(q-p, r-q-e+p) \sum_{k=0}^{n-2} M_{\tau}^{k}\right)\left(\begin{array}{l}
1 \\
1
\end{array}\right)
$$

Proof Note that if we apply any substitution $\phi$ with incidence matrix $M_{\phi}$ to a word $u$ containing $x$ zeros and $y$ ones, then the number of zeros and ones in $\phi(u)$ is given by the elements of $(x, y) M_{\phi}$, respectively. By definition $v_{1}$ has $p$ zeros and $e-p$ ones left of the underlined letter. After applying $\tau^{n-1}$ to $v_{1}$ these zeros and ones yield $(p, e-p) M_{\tau}^{n-1}\left(\begin{array}{l}1 \\ 1\end{array}\right)$ letters left of the underlined letter. Furthermore, $\tau(\underline{0})$ has by definition $q$ zeros and $r-q$ ones left of the underlined letter. Hence $\tau^{n-1}(\underline{0})$ has $(q, r-q)\left(M_{\tau}^{0}+M_{\tau}^{1}+\cdots+M_{\tau}^{n-2}\right)\left(\begin{array}{l}1 \\ 1\end{array}\right)$ letters left of the underlined letter. We see that the total number of letters left of the underlined letter in the central word $\tau^{n-1}\left(v_{1}\right)$ equals

$$
\left((p, e-p) M_{\tau}^{n-1}+(q, r-q) \sum_{k=0}^{n-2} M_{\tau}^{k}\right)\left(\begin{array}{l}
1 \\
1
\end{array}\right) .
$$

From this the result follows directly.

\section{Proposition 5.7}

$$
d(f-p-e g)=q-p
$$

Proof First assume $g=0$ (i.e. $M_{\sigma}$ is an upper matrix). We know from Lemma 5.1 that $f-p$ equals 0 or 1 .

If $f=p$, it follows that $r=e$. Since $v_{1}$ and $\tau(0)$ are both strongly balanced, have the same number of zeros, the same number of ones, and the same number of letters left of the underlined letter, we get $v_{1}=\tau(0)$, hence $q=p$.

Assume $f-p=1$. Then $r=e+b$. Hence $\tau(0)$ is the cyclic shift of $v_{1}$ that you get by cyclically shifting over $b$ positions to the left. Since $b(a+b-(c+d))=$ 
$(b-d)(a+b)+1$, it follows from Lemma 3.5 that the prefix of length $b$ of $\tau(0)$ contains exactly $b-d$ ones, hence exactly $d$ zeros. Thus $q-p=d$.

Now assume $g>0$, hence $M_{\sigma}$ is a lower matrix. We know from Proposition 5.4 that $f-p-e g \leq g+1$. Since the first jump in $w_{\sigma(0)}$ is to the right of length $b$, we see that $w_{\sigma(0)}$ has at most $a-1$ numbers left of 0 , hence $e<a$. It follows that $r<a+b(g+1)$.

Note that

$$
M_{\sigma}^{2}=\left(\begin{array}{cc}
a^{2}+b c+a b g & a b+b d+b^{2} g \\
a c+a^{2} g+c d+b c g+a d g+a b g^{2} & b c+a b g+d^{2}+2 b d g+b^{2} g^{2}
\end{array}\right) .
$$

It follows from Lemma 3.1 and Lemma 4.5 that $w_{1}(-1)=c+d$ and $w_{2}(-1)=$ $a c+c d+a d g+b c+d^{2}+b d g$.

Let $s$ be the positive integer such that $w_{2}(-s)=c+d$. Since $w_{2}(-1) \equiv\left(a^{2}+b c+\right.$ $a b g)^{-1}\left(\bmod \left|w_{2}\right|\right)$ we see that $s \equiv(c+d)\left(a^{2}+b c+a b g\right) \equiv a+b g\left(\bmod \left|w_{2}\right|\right)$ according to Lemma 5.3 (ii). Hence $s=a+b g$.

A calculation gives $b w_{2}(1)=b\left(\left|w_{2}\right|-w_{2}(-1)\right)=(b-d)\left|w_{2}\right|+a+b$. Using a similar argument as in the proof of Lemma 3.5, we see that every subword of $b$ letters in $v_{2}$ contains $d$ zeros and $b-d$ ones, provided that the number in $w_{2}$ at the position directly right of this subword is at least $a+b$. The numbers smaller than $a+b$ in $w_{2}$ are exactly the numbers in $w_{\sigma(0)}$, and since according to Lemma 4.6 their order is preserved in $w_{2}$, the first number left of 0 in $w_{2}$ that is smaller than $a+b$ is $w_{\sigma(0)}(-1)=c+d$ which can be found at position $-s=-(a+b g)$ in $w_{2}$.

Since $r<a+b(g+1)$, the position of the left most letter of $\tau(0)$ is larger than $-(a+b(g+1))$, and therefore the values of $w_{2}$, at the negative positions of $\tau(0)$ are each at least $a+b$. Hence each subword of length $b$ of $\tau(0)$ left of position -1 contains exactly $d$ zeros. Since $r-e=b(f-p-e g)$, we obtain $q-p=d(f-p-e g)$.

The next theorem says that if $\sigma(0)$ is not a Christoffel word, the words $v_{n}$ are generated by another Sturmian substitution. In case $\sigma$ is a Christoffel substitution, this was proved in [15] Theorem 6.1. Recall that $\tau$ is the substitution that has

$$
M_{\tau}:=\left(\begin{array}{cc}
1 & g+1 \\
1 & g
\end{array}\right) M_{\sigma}^{T}\left(\begin{array}{cc}
-g & g+1 \\
1 & -1
\end{array}\right)
$$

as incidence matrix, and which is such that

- if we cyclically shift $\tau(0)$ over $r$ positions to the right, we get a Christoffel word,

- the $(r+1)$ th letter of $\tau(0)$ is underlined,

- $\tau(1)$ is a prefix of $\tau(0)$.

Theorem 5.8 Let $\sigma$ be a primitive Sturmian substitution that has an incidence matrix with determinant 1 , that has a fixed point starting with 0 , and for which $\sigma(0)$ is not a Christoffel word. Let the central words $v_{n}$ for $n \geq 1$ be defined as before. Then $v_{n}=\tau^{n-1}\left(v_{1}\right)$. 
Proof Note that

$$
\begin{gathered}
M_{\sigma}=\left(\begin{array}{ll}
1 & 0 \\
g & 1
\end{array}\right)\left(\begin{array}{ll}
a & b \\
c & d
\end{array}\right) \text { and } \\
M_{\tau}=\left(\begin{array}{ll}
1 & 1 \\
1 & 0
\end{array}\right)\left(\begin{array}{ll}
1 & g \\
0 & 1
\end{array}\right)\left(\begin{array}{ll}
a & c \\
b & d
\end{array}\right)\left(\begin{array}{cc}
0 & 1 \\
1 & -1
\end{array}\right) .
\end{gathered}
$$

Hence for $k>0$

$$
\begin{gathered}
(1,0) M_{\sigma}^{k}\left(\begin{array}{l}
e \\
f
\end{array}\right)=(e, f)\left[\left(\begin{array}{ll}
a & c \\
b & d
\end{array}\right)\left(\begin{array}{ll}
1 & g \\
0 & 1
\end{array}\right)\right]^{k}\left(\begin{array}{l}
1 \\
0
\end{array}\right) \\
=(e, f)\left(\begin{array}{ll}
a & c \\
b & d
\end{array}\right)\left[\left(\begin{array}{ll}
1 & g \\
0 & 1
\end{array}\right)\left(\begin{array}{ll}
a & c \\
b & d
\end{array}\right)\right]^{k-1}\left(\begin{array}{l}
1 \\
0
\end{array}\right) \\
=(e, f)\left(\begin{array}{ll}
a & c \\
b & d
\end{array}\right)\left(\begin{array}{cc}
0 & 1 \\
1 & -1
\end{array}\right) \\
{\left[\left(\begin{array}{ll}
1 & 1 \\
1 & 0
\end{array}\right)\left(\begin{array}{ll}
1 & g \\
0 & 1
\end{array}\right)\left(\begin{array}{ll}
a & c \\
b & d
\end{array}\right)\left(\begin{array}{cc}
0 & 1 \\
1 & -1
\end{array}\right)\right]^{k-1}\left(\begin{array}{l}
1 \\
1
\end{array}\right)} \\
=(e, f)\left(\begin{array}{ll}
c & a-c \\
d & b-d
\end{array}\right) M_{\tau}^{k-1}\left(\begin{array}{l}
1 \\
1
\end{array}\right) .
\end{gathered}
$$

By Proposition 5.7 and $r=e+b(f-p-e g)$ we have

$$
(f-p-e g)(d, b-d)=(q-p, r-q-e+p) .
$$

Hence

$$
\begin{aligned}
(e, f)\left(\begin{array}{ll}
c & a-c \\
d & b-d
\end{array}\right) & =(e, p)\left(\begin{array}{ll}
1 & g \\
0 & 1
\end{array}\right)\left(\begin{array}{ll}
c & a-c \\
d & b-d
\end{array}\right)+(0, f-p-e g)\left(\begin{array}{ll}
c & a-c \\
d & b-d
\end{array}\right) \\
& =(p, e-p) M_{\tau}+(q-p, r-q-e+p) .
\end{aligned}
$$

From the above formulas we conclude that for $n \geq 1$

$$
\begin{aligned}
& (1,0) \sum_{k=0}^{n-1} M_{\sigma}^{k}\left(\begin{array}{l}
e \\
f
\end{array}\right) \\
& =\left((p, e-p) \sum_{k=0}^{n-1} M_{\tau}^{k}+(q-p, r-q-e+p) \sum_{k=0}^{n-2} M_{\tau}^{k}\right)\left(\begin{array}{l}
1 \\
1
\end{array}\right)
\end{aligned}
$$

According to Lemma 5.5, the expression on the left represents the number of letters left of the underlined letter in $v_{n}$, and according to Lemma 5.6 the expression on 
the right represents the number of letters left of the underlined letter in $\tau^{n-1}\left(v_{1}\right)$. Since $v_{n}$ and $\tau^{n-1}\left(v_{1}\right)$ are strongly balanced words, and they contain according to Theorem 5.4 of [15] the same number of zeros and ones, the result follows.

\section{Final remarks}

If the substitution $\tau$ has a fixed point starting with 0 , we can apply the procedure of projecting the cutting paths corresponding to $\tau$, to construct the substitution $\phi$, in the same way as we constructed the substitution $\tau$ from the substitution $\sigma$. It is easy to check that if $M_{\sigma}$ is an upper matrix then $M_{\phi}=M_{\sigma}$, hence $\phi(0), \phi(1)$ are cyclic shifts of $\sigma(0), \sigma(1)$ respectively. We have seen in [15] Corollary 6.2 that if $\sigma$ is a Christoffel substitution, then we get $\phi=\sigma$. This is not the case in general, as the following example shows.

Example 4 Let $M_{\sigma}=\left(\begin{array}{ll}3 & 4 \\ 2 & 3\end{array}\right)$ and $\sigma(0)=0101101, \sigma(1)=01101$. Then $e=1$, $f=1, p=0$ and $r=5$. We get $M_{\tau}=\left(\begin{array}{ll}5 & 2 \\ 2 & 1\end{array}\right)$ and $\tau(0)=0100100, \tau(1)=010$. Repeating this process, we get $e=4, f=1, p=1$ and $r=4$, which results in $\phi(0)=1011010, \phi(1)=10110$.

If $\sigma$ is a Sturmian substitution that has an incidence matrix with determinant 1 , for which $\sigma(0)$ is a Christoffel word, but that is not a Christoffel substitution, there need not exist a substitution $\tau$ such that $v_{n}=\tau^{n-1}\left(v_{1}\right)$, as the following example shows.

Example 5 Let $M_{\sigma}=\left(\begin{array}{ll}1 & 1 \\ 1 & 2\end{array}\right)$ and $\sigma(0)=01, \sigma(1)=101$. Then we get the following table for $v_{n}$.

\begin{tabular}{c|ccccccccccccc}
$n$ & \multicolumn{11}{c|}{$v_{n}$} \\
\hline 1 & & & & & $\underline{0}$ & 1 & & & & & & & \\
2 & & & & 1 & $\underline{0}$ & 0 & 1 & 0 & & & & & \\
3 & 0 & 1 & 0 & 1 & $\underline{0}$ & 0 & 1 & 0 & 0 & 1 & 0 & 1 & 0
\end{tabular}

It is clear that there is no substitution $\tau$ such that $\tau\left(v_{1}\right)=v_{2}$ and $\tau\left(v_{2}\right)=v_{3}$.

If $\sigma$ is a Sturmian substitution with a fixed point starting with 0 that has an incidence matrix $M_{\sigma}$ with determinant -1 , we can still form the central words $v_{n}$, except that for odd $n$, we need to reflect the central progressions $w_{n}$ in the origin, before we construct $v_{n}$ from $w_{n}$. Since the substitution $\sigma^{2}$ has incidence matrix with determinant 1 , it is clear that there exists a substitution $\tau_{2}$ such that $v_{2 n}=\tau_{2}\left(v_{2 n-2}\right)$. But as the following example shows, there does not need to exist a substitution $\tau$ such that $v_{n}=\tau\left(v_{n-1}\right)$.

Example 6 Let $M_{\sigma}=\left(\begin{array}{ll}2 & 1 \\ 1 & 0\end{array}\right)$ and $\sigma(0)=001, \sigma(1)=0$. Then we get the following table for $v_{n}$. 


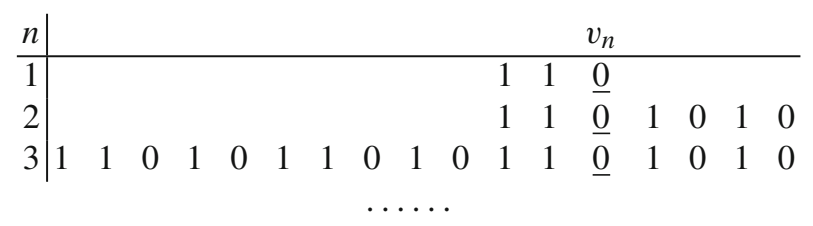

It is easy to check that there is no substitution $\tau$ such that $\tau\left(v_{2}\right)=v_{3}$. However, this example suggests that if we define the substitution $\tau$ by $\tau(\underline{0})=11 \underline{0}, \tau(1)=10$, then we have $v_{n}=\tau\left(\overline{v_{n-1}}\right)$, where we denote by $\bar{u}$ the word $u$ mirrored in the origin. An interesting question is if this holds for all Sturmian substitutions that have an incidence matrix with determinant -1 .

Acknowledgments We like to thank Robert Tijdeman and the referee for valuable remarks and suggestions.

Open Access This article is distributed under the terms of the Creative Commons Attribution Noncommercial License which permits any noncommercial use, distribution, and reproduction in any medium, provided the original author(s) and source are credited.

\section{References}

1. Arnoux, P., Ito, S.: Pisot Substitutions and Rauzy fractals. Bull. Belg. Math. Soc. 8, 181-207 (2001)

2. Arnoux, P., Ito, S., Sano, Y.: Higher dimensional extensions of substitutions and their dual maps. J. Anal. Math. 83, 183-206 (2001)

3. Borel, J.-P., Laubie, F.: Constructions de mots de Christoffel. C. R. Acad. Sci. Paris (I) 313, 483-485 (1991)

4. Borel, J.-P., Laubie, F.: Quelques mots sur la droite projective réelle. J. Théor. Nombres Bordeaux 5, 23-51 (1993)

5. Canterini, V., Siegel, A.: Geometric Representations of Substitutions of Pisot type. Trans. Am. Math. Soc. 353, 5121-5144 (2001) (electronic)

6. Christoffel, E.B.: Observatio arithmetica. Math. Ann. 6, 145-152 (1875)

7. Fuchs, C., Tijdeman, R.: Substitutions, abstract number systems and the space filling property. Ann. Inst. Fourier (Grenoble) 56, 2345-2389 (2006)

8. Ito, S., Rao, H.: Purely periodic $\beta$-expansions with Pisot unit base. Proc. Am. Math. Soc. 133 (2005), 953-964 (electronic)

9. Lothaire, M.: Algebraic Combinatorics on Words. Cambridge University Press, London (2002)

10. Laubie, F.: Prolongements homographiques de substitutions de mots de Christoffel. C. R. Acad. Sci. Paris (I) 313, 565-567 (1991)

11. Morse, M., Hedlund, G.A.: Symbolic dynamics II: Sturmian trajectories. Am. J. Math. 62, 1-42 (1940)

12. Pytheas Fogg, N.: Substitutions in Dynamics, Arithmetics and Combinatorics. Springer, Heidelberg (2002)

13. Rauzy, G.: Nombres algébraiques et substitutions. Bull. Soc. Math. France 110, 147-178 (1982)

14. Richome, G.: Test-words for Sturmian morphisms. Bull. Belg. Math. Soc. 6, 481-489 (1999)

15. Rosema, S.W.: Substitutions on two letters, cutting segments and their projections. J. Théor. Nombres Bordeaux 19, 523-545 (2007)

16. Rosema, S.W., Tijdeman, R.: The tribonacci substitution, integers, electron. J. Combin. Number Th. $\mathbf{5}(3), \mathrm{A} 13(2005)$

17. Séébold, P.: Fibonacci morphisms and Sturmian words. Theor. Comput. Sci. 195, 91-109 (1991)

18. Series, C.: The geometry of Markoff numbers. Math. Intelligencer 7(3), 20-29 (1985) 